\title{
Gene expression of inflammasome components in peripheral blood mononuclear cells (PBMC) of vascular patients increases with age
}

\author{
Xiaoyu Wu ${ }^{1,2}$, Maani Hakimi ${ }^{1,3}$, Markus Wortmann ${ }^{1}$, Jian Zhang ${ }^{2}$, Dittmar Böckler ${ }^{1}$ and Susanne Dihlmann ${ }^{1 *}$
}

\begin{abstract}
Background: Chronic low-grade inflammation is considered a driver of many age-related disorders, including vascular diseases (inflammaging). Inhibition of autophagic capacity with ageing was postulated to generate a pro-inflammatory condition via activation of inflammasomes, a group of Interleukin-1 activating intracellular multi-protein complexes. We thus investigated gene expression of inflammasome components in PBMC of 77 vascular patients (age 22-82) in association with age.

Findings: Linear regression of real-time qRT-PCR data revealed a significant positive association of gene expression of each of the inflammasome components with age (Pearson correlation coefficients: AIM2: $r=0.245 ; P=0.032$; NLRP3: $r=0.367 ; P=0.001 ;$ ASC (PYCARD): $r=0.252 ; P=0.027$; CASP1: $r=0.296 ; P=0.009 ;$ CASP5: $r=0.453 ; P=0.00003$; ILIB: $r=0.247 ; P=0.030$ ). No difference in gene expression of AIM2, NLRP3, ASC CASP1, and CASP5 was detected between PBMC of patients with advanced atherosclerosis and other vascular patients, whereas IL1B expression was increased in PBMC of the latter group $(P=0.0005)$.
\end{abstract}

Conclusion: The findings reinforce the systemic pro-inflammatory phenotype reported in elderly by demonstrating an increased phase-1 activation of inflammasomes in PBMC of vascular patients.

Keywords: Vascular disease, Inflammation, Aging, AIM2, NLRP3, Atherosclerosis

\section{Findings}

\section{Background}

The concept of inflammaging implies that a low-grade pro-inflammatory status, predisposing the organism to chronic diseases, appears during the ageing process [1]. A number of reports have provided experimental evidence for this hypothesis and remodeling of the immune system was postulated to drive many age-related disorders [2]. In addition, altered function of innate immune cells, in particular changes in monocytes and macrophages, have been described in mice [3] and humans [4]. Clinically, aging is associated with increases of circulating IL-18 and IL-6 plasma levels, whereas IL-1 $\beta$ is undetectable $[5,6]$.

During aging, injuries, necrotic cell death and metabolic cell stress accumulate thereby increasing the risk

\footnotetext{
* Correspondence: susanne.dihlmann@med.uni-heidelberg.de

${ }^{1}$ Department of Vascular and Endovascular Surgery, University of Heidelberg, Im Neuenheimer Feld 110, 69120 Heidelberg, Germany

Full list of author information is available at the end of the article
}

of chronic inflammation [7, 8]. Dead cells and their metabolites are usually cleared by autophagy though macrophages. A disturbed interplay between autophagy and the inflammasomes was recently postulated to link inflammaging with vascular and other pathologies [9]. The inflammasomes are a family of multiprotein complexes for danger signal recognition that are induced by pathogens or cell debris (damage associated molecular patterns, DAMPS) $[10,11]$. Expression levels of the inflammasome components are generally low and require a two phase induction: A priming phase stimulates gene expression of inactive precursors before a second signal can stimulate assembly of the multiprotein complex consisting of sensor proteins, the adaptor protein apoptosis-associated specklike protein with a caspase activation and recruitment domain (ASC) and inflammatory caspases (Caspase-1 or Caspase-5). Upon assembly, the caspases are enzymatically cleaved by the complex resulting in their active form, which then stimulates enzymatic cleavage of Interleukin$1 \beta$ (IL-1 $\beta$ ) and IL-18 from inactive precursors [10]. 
Depending on the initial sensor, several subfamilies are distinguished: The NOD-like receptors (NLR), including NLRP3 and others, act as sensors for intracellular damage associated signals such as cholesterol crystals, nanoparticles, reactive oxygen species and others. The DNA sensors, including Absent in Melanoma 2 (AIM2) act as sensors for intracellular dsDNA [10].

Given the recently demonstrated involvement of inflammasome activation in vascular disease [12-14] and its putative role in inflammaging, we here addressed gene expression of inflammasome components in PBMC of vascular patients in association with age.

\section{Materials and methods}

Venous blood was taken from 77 vascular patients on the day of their hospitalization according to the standard operating procedures of the Vascular Biobank Heidelberg (VBBH). All patients gave their written informed consent to the study, which was approved by the ethical committee of the University of Heidelberg (S-301/2013 and S-412/2013). Patients' characteristics are described in Additional file 1: Table S1.

PBMC and plasma were separated according to standard procedures (for details see Additional file 2), RNA extraction and reverse transcription were performed as described earlier ([13] and Additional file 2). Quantitative analysis of gene expression was performed by real time PCR and relative expression was determined by using individual standard amplification curves of each transcript relative to the corresponding mean expression of three reference transcripts (GAPDH plus B2M plus $A C T B$ ).

Active, cleaved IL-1 $\beta$ (p17) in plasma was quantified by ELISA, Caspase-1 protein was detected by Western blotting (for details see Additional file 2).

\section{Results and discussion}

As shown in Fig. 1, best fit regression revealed a close association of AIM2, NLRP3, ASC, CASP1, CASP5, and $I L 1 B$ gene expressions with age of the patients. The strongest correlation was found for $C A S P 5$ with a correlation coefficient of $0.468(P=0.00003)$. Regression coefficients and p-values of the linear regression analysis are summarized in Table 1.

To examine, whether the increased expression of inflammasome components resulted in increased activation of the inflammasome multiprotein complex, we next determined the amount of its effector proteins, activated caspase-1 (p10) and activated Il-1 $\beta$ (p17). Active IL- $1 \beta$ was detected in plasma of 3 out of 30 patients with abdominal aortic aneurysm and in plasma derived from one patient

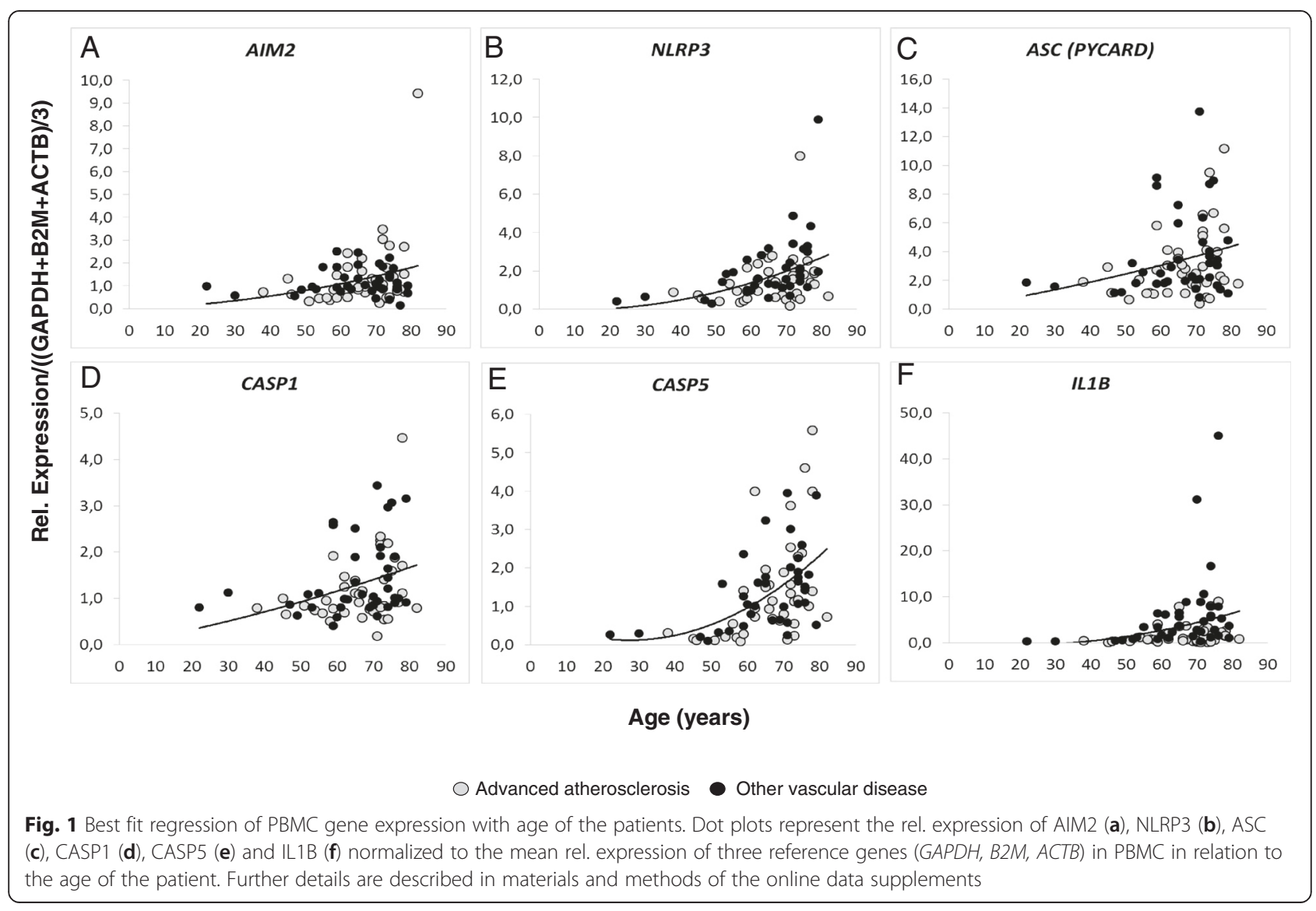


Table 1 Linear regression analysis of gene expression with age of the patients

\begin{tabular}{lllll}
\hline & $\begin{array}{l}\text { Pearson correlation } \\
\text { Coefficient }\end{array}$ & $\begin{array}{l}\text { Standard } \\
\text { Error }\end{array}$ & $95 \% \mathrm{Cl}$ & P-value \\
\hline AIM2 & 0.245 & 0.074 & $0.094-0.369$ & 0.032 \\
NLRP3 & 0.367 & 0.054 & $0.254-0.477$ & 0.001 \\
ASC(PYCARDN & 0.252 & 0.080 & $0.114-0.412$ & 0.027 \\
CASP1 & 0.296 & 0.089 & $0.116-0.483$ & 0.009 \\
CASP5 & 0.453 & 0.069 & $0.320-0.570$ & 0.00003 \\
IL1B & 0.247 & 0.373 & $0.150-0.373$ & 0.030 \\
\hline
\end{tabular}

with an aortic dissection (data not shown). Western blotting of PBMC lysates showed cleaved, active caspase-1 (p10) together with pro-Caspase-1 (p50) in all samples (Example in Fig. 2a). Neither the IL-1 $\beta$ plasma levels nor the ratio of Caspase- $1 \mathrm{p} 10 / \mathrm{p} 10+\mathrm{p} 50$, indicating relative activity of the inflammasome complex, were associated with age (data not shown).

To adjust for vascular diseases, such as atherosclerosis, that might interfere with inflammasome activation [12, 15], inflammasome gene expression and activities in the group of patients with advanced atherosclerosis (carotid stenosis, peripheral artery disease and arterial stenosis; $n=39$ ) were compared with those derived from patients with other vascular disease (aortic aneurysms, other; $n=38$ ). As shown in Fig. 2, AIM2, NLRP3, ASC, CASP1, and CASP5 gene expressions did not differ between the groups (Fig. 2b-f). In contrast, $I L 1 B$ gene expression was significantly higher in the control group compared to the atherosclerosis group $(P=0.0005$; Fig. $2 \mathrm{~g}$ and Additional file 1: Table S1). This unexpected finding may be ascribed to the composition of the control group which contained many samples from patients with aortic aneurysms. Locally increased gene

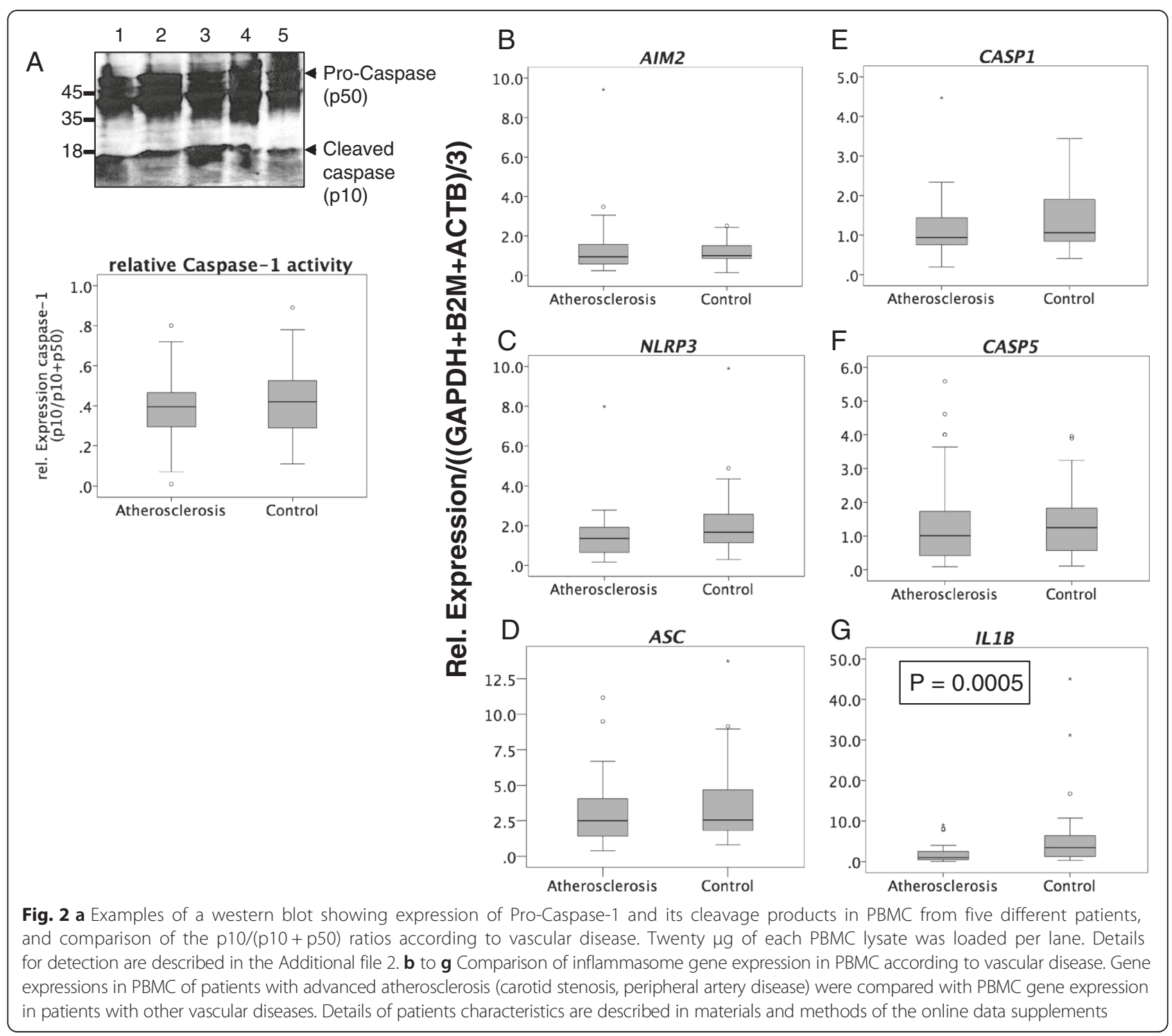


expression of $I L 1 B$ is known to be increased in AAA tissue samples [16]. Whether PBMC from AAA-patients do also express increased levels of IL1B and other inflammasome components is currently under our investigation and will be published in a separate study. No difference in the relative levels of active Caspase-1 (p10) could be detected between the groups (Fig. 2a and Additional file 1: Table S1).

Despite some indications from animal experiments [17], it is currently unknown, whether systemic inflammasome activation is associated with human ageing. Our data present clear evidence for this hypothesis by demonstrating, that gene expression of AIM2, NLRP3, ASC, CASP1, CASP5, and IL1B in PBMC of vascular patients increases with age. Future studies on inflammasome gene expression of PBMC in healthy people of different ages will be necessary to demonstrate whether this phenomenon applies to ageing in general, although the definition of "healthy" might be difficult in individuals above 70 years.

Regardless of the limitation to vascular patients, our findings reinforce results from previous studies, describing age-associated mechanisms that are required for priming of inflammasome components. For example, constitutive transcriptional activity of NF- $\mathrm{kB}$, which is necessary for induction of NLRP3 and ILIB gene expression, has been demonstrated in aged tissues and organisms [18-20]. In addition, increased levels of the AIM2 protein in human fibroblasts were associated with senescence and increased production of IL-1 $\beta$ [21].

Since phase- 1 activation was not accompanied by increased IL-1 $\beta$ and Caspase-p10 levels in our study, we conclude that full inflammasome assembly and activation, requiring a second signal [10], do not occur with a higher frequency in PBMC of the elderly. This is in line with previous reports, demonstrating that PBMC of young and older individuals do not differ in Il- $1 \beta$ release after stimulation with LPS in vitro [4].

Instead, our data point to an age-associated shift towards a preactivated first line of defense by innate immunity. Given a systemic increase in priming of inflammasome genes through constitutive gene expression in PBMC with ageing, the threshold for full inflammasome activation by the second signal derived from local DAMP recognition in different tissues might be reached earlier and predispose individuals to vascular and other chronic diseases. Cummulative evidence supports the existence of such ageassociated changes in the cellular components of the innate immune system $[2,4]$. However, as yet, we cannot deduce from our data, whether the increased inflammasome gene expression results from a general activation across all PBMC or from an altered PBMC composition, i.e. an increase in monocyte numbers, as reported previously [22]. Further investigations, addressing this question are currently under examination. In addition, our ongoing studies aim to elucidate the interaction of primed PBMC with vascular tissues and their impact on vascular disease progression. The reason for the increased gene expression of inflammasome components in PBMC during ageing is unknown so far. Besides DAMPS and pathogens serving as immunomodulators, the sympathethic nervous system was recently described to affect the functions of both innate and adaptive immune cells via the $\beta$-adrenergic receptor ( $\beta$-AR) $[23,24]$. It remains to be shown, whether ageing-associated changes in $\beta$-AR function contribute to altered inflammasome gene expression in PBMC.

In summary, the present study adds important findings to the concept of inflammaging by demonstrating an agedependent increase in activation of innate immunity via systemic priming of inflammasomes in vascular patients.

\section{Additional files}

Additional file 1: Table S1. Comparison of age, sex and gene expression in PBMC from patients with advanced atherosclerosis and PBMC from other vascular patients. (DOCX $16 \mathrm{~kb}$ )

Additional file 2: Materials and methods. (DOCX $24 \mathrm{~kb}$ )

\section{Abbreviations}

AIM2: Absent in Melanoma 2; ASC: Apoptosis-associated speck-like protein with a caspase activation and recruitment domain; CASP: Caspase; DAMP: Damage-associated molecular pattern; IL: Interleukin; IL-1ß: Interleukin1ß; IL-18: Interleukin-18; IL-6: Interleukin-6; NLRP3: NOD-like receptor protein 3; PBMC: Peripheral blood mononuclear cells.

\section{Competing interests}

The authors declare that they have no competing interests.

\section{Authors' contributions}

XW carried out the PBMC separation, gene expression analysis and statistical analysis. $\mathrm{MH}$ and MW participated in sampling, tissue banking and interpretation of the results. JZ and DB helped to draft the manuscript. SD conceived of the study, designed the experiments, helped with the statistical analysis and Western blotting, and drafted the manuscript. All authors read and approved the final manuscript.

\section{Acknowledgements}

We thank Anja Spieler for excellent technical assistance and Sven Schnaidt for statistical counseling. The study was funded by an Oversea Study Fellowship from the China Scholarship Council to Xiaoyu Wu. We acknowledge financial support by the Department of Vascular and Endovascular surgery of the University of Heidelberg, Germany.

\section{Author details}

'Department of Vascular and Endovascular Surgery, University of Heidelberg, Im Neuenheimer Feld 110, 69120 Heidelberg, Germany. 'Department of Vascular \& Thyroid Surgery, The First Hospital of China Medical University, Shenyang, China. ${ }^{3}$ Vaskuläre Biomaterialbank Heidelberg, VBBH, Heidelberg, Germany.

Received: 27 July 2015 Accepted: 23 September 2015 Published online: 06 October 2015

\section{References}

1. Franceschi C, Bonafè M, Valensin S, Olivieri F, De Luca M, Ottaviani $E_{\text {, et al. }}$ Inflamm-aging. An evolutionary perspective on immunosenescence. Ann N Y Acad Sci. 2000;908:244-54.

2. Larbi A, Franceschi C, Mazzatti D, Solana R, Wikby A, Pawelec G. Aging of the immune system as a prognostic factor for human longevity. Physiology (Bethesda). 2008;23:64-74. 
3. Kovacs EJ, Palmer JL, Fortin CF, Fülöp Jr T, Goldstein DR, Linton PJ. Aging and innate immunity in the mouse: impact of intrinsic and extrinsic factors Trends Immunol. 2009;30(7):319-24.

4. Pinke KH, Calzavara B, Faria PF, do Nascimento MP, Venturini J, Lara VS Proinflammatory profile of in vitro monocytes in the ageing is affected by lymphocytes presence. Immun Ageing. 2013;10(1):22.

5. Ferrucci L, Corsi A, Lauretani F, Bandinelli S, Bartali B, Taub DD, et al. The origins of age-related proinflammatory state. Blood. 2005;105(6):2294-9.

6. Dinarello CA. Interleukin-1 beta and the autoinflammatory diseases. N Engl J Med. 2009:360(23):2467-70.

7. Feldman, N., A. Rotter-Maskowitz, and E. Okun, DAMPs as mediators of sterile inflammation in aging-related pathologies. Ageing Res Rev, 2015. doi: 10.1016/j.arr.2015.01.003.

8. Goldberg R, Prescott N, Lord GM, MacDonald TT, Powell N. The unusual suspects-innate lymphoid cells as novel therapeutic targets in IBD. Nat Rev Gastroenterol Hepatol. 2015;12(5):271-83.

9. Salminen A, Kaarniranta K, Kauppinen A. Inflammaging: disturbed interplay between autophagy and inflammasomes. Aging (Albany NY). 2012;4(3):166-75.

10. Latz E, Xiao TS, Stutz A. Activation and regulation of the inflammasomes. Nat Rev Immunol. 2013;13(6):397-411.

11. Kono H, Kimura $Y$, Latz E. Inflammasome activation in response to dead cells and their metabolites. Curr Opin Immunol. 2014;30:91-8.

12. Duewell $\mathrm{P}$, Kono H, Rayner KJ, Sirois CM, Vladimer G, Bauernfeind FG, et al. NLRP3 inflammasomes are required for atherogenesis and activated by cholesterol crystals. Nature. 2010;464(7293):1357-61.

13. Dihlmann S, Erhart P, Mehrabi A, Nickkholgh A, Lasitschka F, Böckler D, et al. Increased expression and activation of absent in melanoma 2 inflammasome components in lymphocytic infiltrates of abdominal aortic aneurysms. Mol Med. 2014;20(1):230-7.

14. Hakimi M, Peters A, Becker A, Böckler D, Dihlmann S. Inflammation-related induction of absent in melanoma 2 (AIM2) in vascular cells and atherosclerotic lesions suggests a role in vascular pathogenesis. J Vasc Surg. 2014;59(3):794-803. e2.

15. Samstad EO, Niyonzima N, Nymo S, Aune MH, Ryan L, Bakke SS, et al. Cholesterol crystals induce complement-dependent inflammasome activation and cytokine release. J Immunol. 2014;192(6):2837-45.

16. Golledge AL, Walker P, Norman PE, Golledge J. A systematic review of studies examining inflammation associated cytokines in human abdominal aortic aneurysm samples. Dis Markers. 2009;26(4):181-8.

17. Youm YH, Grant RW, McCabe LR, Albarado DC, Nguyen KY, Ravussin A, et al. Canonical Nlrp3 inflammasome links systemic low-grade inflammation to functional decline in aging. Cell Metab. 2013;18(4):519-32.

18. Spencer NF, Poynter ME, Im SY, Daynes RA. Constitutive activation of NF-kappa B in an animal model of aging. Int Immunol. 1997;9(10):1581-8

19. Xiao ZQ, Majumdar AP. Induction of transcriptional activity of AP-1 and NF-kappaB in the gastric mucosa during aging. Am J Physiol Gastrointest Liver Physiol. 2000;278(6):G855-65.

20. Helenius M, Kyrylenko S, Vehviläinen P, Salminen A. Characterization of aging-associated up-regulation of constitutive nuclear factor-kappa B binding activity. Antioxid Redox Signal. 2001;3(1):147-56.

21. Duan X, Ponomareva L, Veeranki S, Panchanathan R, Dickerson E, Choubey D. Differential Roles for the Interferon-Inducible IFI16 and AIM2 Innate Immune Sensors for Cytosolic DNA in Cellular Senescence of Human Fibroblasts. Mol Cancer Res. 2011;9(5):589-602.

22. Della Bella $S$, Bierti $L$, Presicce $P$, Arienti $R$, Valenti $M$, Saresella $M$, et al. Peripheral blood dendritic cells and monocytes are differently regulated in the elderly. Clin Immunol. 2007;122(2):220-8.

23. Lorton $\mathrm{D}$, Bellinger DL. Molecular mechanisms underlying beta-adrenergic receptor-mediated cross-talk between sympathetic neurons and immune cells. Int J Mol Sci. 2015;16(3):5635-65.

24. Santulli G, laccarino G. Pinpointing beta adrenergic receptor in ageing pathophysiology: victim or executioner? Evidence from crime scenes. Immun Ageing. 2013;10(1):10.

\section{Submit your next manuscript to BioMed Central and take full advantage of:}

- Convenient online submission

- Thorough peer review

- No space constraints or color figure charges

- Immediate publication on acceptance

- Inclusion in PubMed, CAS, Scopus and Google Scholar

- Research which is freely available for redistribution 